urban Canadians. The physician must adapt to these The same is true on orbit, with crews generally international composition.

Romanow Commission: "I believe that the success of our Health Care System as a whole will be judged not by the quality or service available in the best urban facilities, but by the equality of service Canada can provide to its remote and northern communities."

Risk management

Living in a remote area is a health risk in itself for example, the chances of surviving a major hea trauma are several orders of magnitude lower when the nearest neurosurgeon and ICU are several hours away by flight. Remoteness also drives the way we organize patient follow-up for more benign ailments: norther physicians tend to err on the conservative side, general, to further minimize risks of complications.

The local population understands and accepts these risks; the challenge for northern health care providers, and the responsibility of the healthcare system, is to ensure these discrepancies are minimized,

Similar concerns apply to on-orbit medical
care. For example, in deciding the content of the onboard medical kit, one must decide what pathologies the crew could likely treat successfully. Deciding whether a particular iliness or injury is survivable or not on-orbit is a matter of ongoing speculation and debate. This uncertainty is essentially what drives the requirement for crewmembers to undergo such stringent medical screening, in the hope of minimizing risk. Again, as we envision deep-space missions wherein medical evacuation is not an option, the listically treat only gets more relevant.

David Saint-Jacques (MD, PhD) is an astronaut candidate with the Canadian Space Agency. He and is currently in basic training at NASA's Johnson Space Center in Houston, Texas. He was selected in 2009 while he was working as a family physician in the Inuti community of Puvirnituq, in Nunavik, Northern Quebec. He eceived a B.Eng in engineering physics from Ecole Polytechnique in Montreal, and worked as a biomedical engineer in Paris, France. He subsequenly oblained a PhD in astrophysics al Cambridge Unisersity, UK and Laval, and completed a residency in family medicine at McGill University.

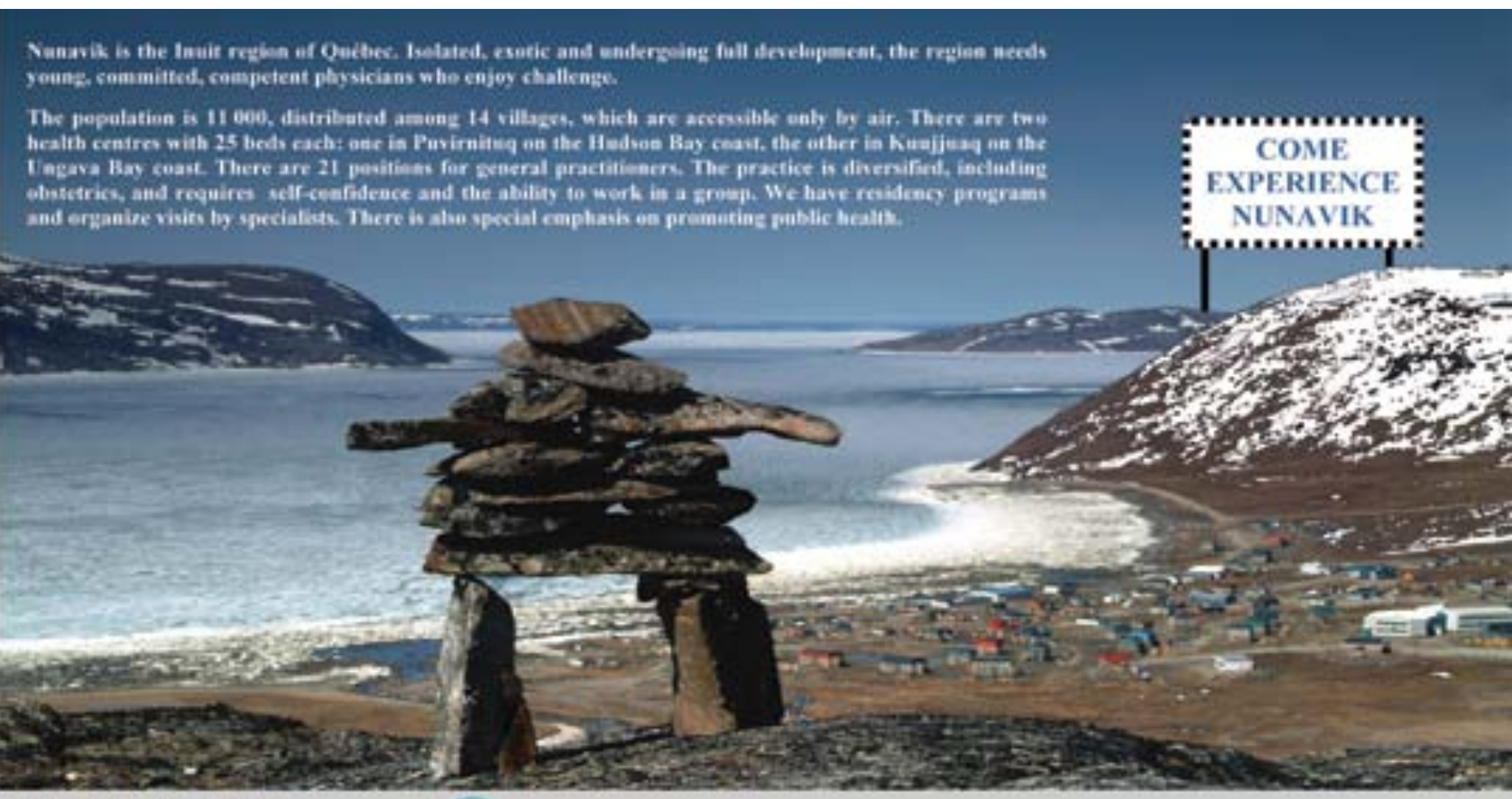

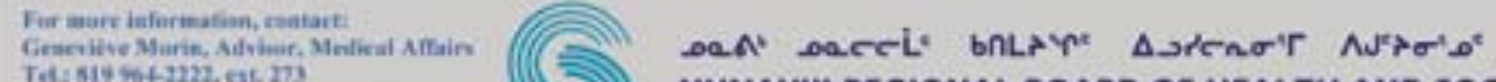

Sini sepvices REGIE RÉGIONALE DE LA SANTE ET DES SERVICES SOCIAUX NUNAVIK
CROSSROADS

\section{Physicians as Astronauts}

Robert Thirsk

In 2009 I had the good fortune to fly on a ong duration space mission. With two crewmates, I launched aboard a Russian Soyuz rocket from the Balkonur Cosmodro Russin Soyz rocket from were traveling at a speed of 28,000 kiloseters per hour through an environment devoid of air water and anything familiar. Two days later we rendezvoused with the International Space Station (ISS) at an altitude of $350 \mathrm{~km}$. As our Soyuz vehicle docked with the Station, we began an incredible space odyssey as members of the ISS Expedition 20/21 crew.

This Expedition marked the first time that the ISS hosted a permanent crew of six. My international crewmates (from Russia, the United States, Japan and Belgium) and I performed an unprecedented amount of multidisciplinary research (Figure 1). We also performed complex robotic erations, spacewalks, and work of Station systems and payloads (Figure 2). Six months later my Soyuz crewmates and I undocked from the Station and landed back in Kazakhstan. During our stay in space, we completed 3,000 orbits of the Earth and traveled 125,000,000 $\mathrm{km}$. It was truly an odyssey.

This ISS expedition as well as my earlier Space Shuttle mission have enriched me in ways I can never fully explain. I often reflect on the career some of my medical colleagues, this path seems some of my medical colleagues, this path seems of medicine have in common with space exploration?".

In the following paragraphs, I describe the astronaut profession and its commonalities with mative experience and the spaceflight environment

*To whom correspondence should be addressed.

Dr. Robert Thirsk Email: robertb thirsk@nasa gov

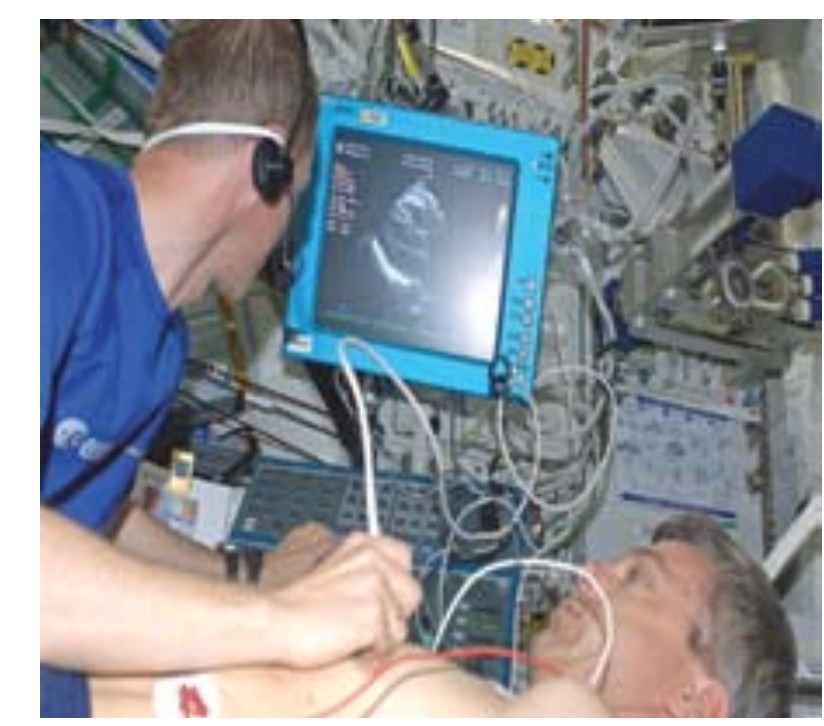

(1)

cardiography on Ro astronaut Frank De Winne performs echotigated cardiovascular adaptation to weightlessness.

is alien to anything in the clinical world. However, a career transition to space exploration after investing so much time and effort in a medical career is not unusual. A well-trained astronaut exhibits many of the same knowledge, skills and professional at tributes of an exemplary physician. Indeed, a medcal background forms an excellent foundation for
career in astronautics.

\section{SELECTION}

An astronaut career begins with selection even more protracted, competitive and rigorous than it is for medical school. The Canadian Space Agency's most recent recruitment campaign in 2008/09 lasted 12 months and saw 5,300 people apply for only two available positions.

Astronaut candidates represent a wide spectrum of professionals such as test pilots, engineers, scientists, educators and physicians. Candidates who have considerable experience working 


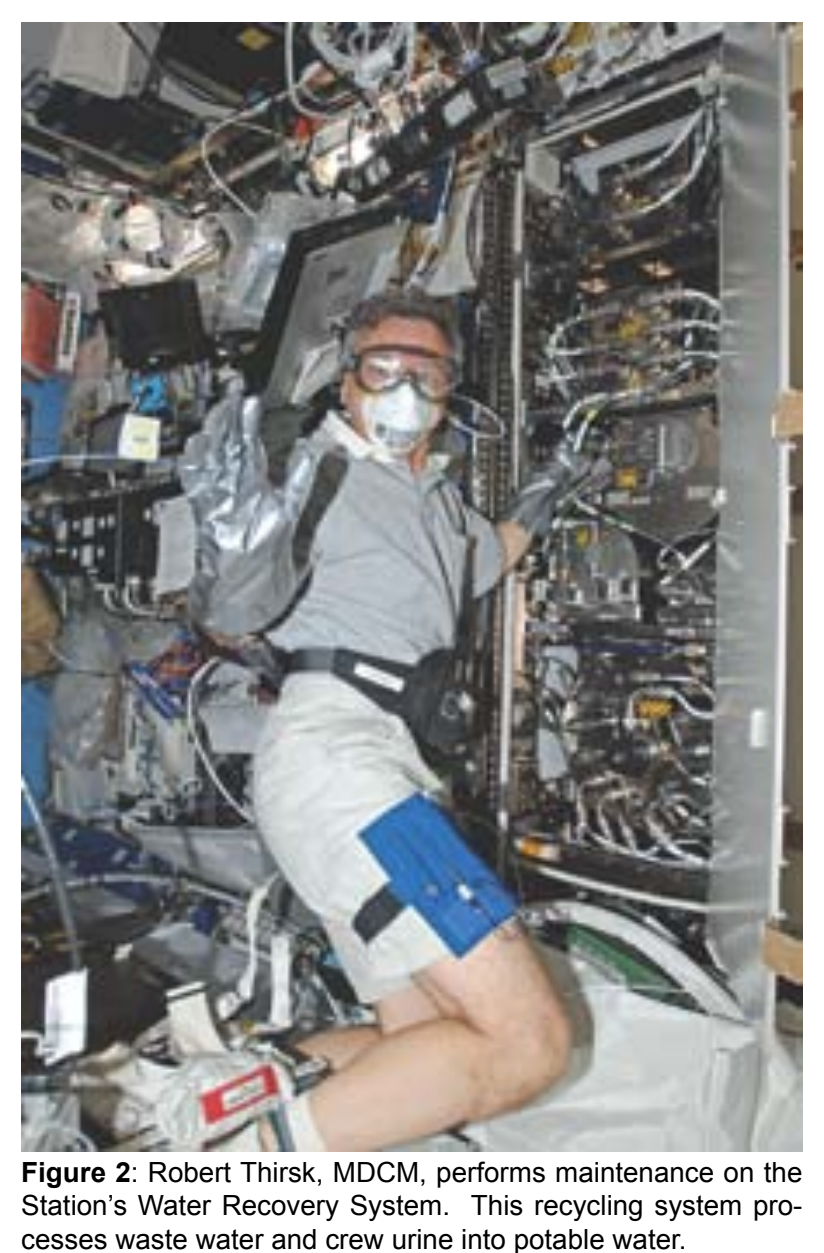

in a team setting and who habitually work outside their comfort zones are highly regarded (Figure 3). Like prospective medical students, astronaut candidates complete questionnaires and submit university transcripts, letters of reference and a personal essay ("why I want to be an astronaut") as part of the application process. During the latter stages of the selection process, a series of interviews are conducted with the selection committee. A very thorough medical evaluation is performed A ve miner mo Eath can become problenside in spaceflight environment.

an astronaut regularly takes us to the limits of our physical, mental and emotional abilities. Accordingly the selection process includes physical fitness, cognitive and psychiatric evaluations. Hours of psychometric tests and psychological interviews ensure that the chosen candidates have the motivation, personalities and support mechanisms to do well in the demanding training and flight environments.

Aptitude tests assess a candidate's team skills, creativity and tolerance to stress. During the most recent recruitment campaign, the candidates potential to learn specialized skills such as robotics, EVA (Extra Vehicular Activity) and foreign languages was also considered.

Finally, interpersonal and communication skills are evaluated. In addition to operational duties, astronauts function as spokespersons for the Canadian Space Agency. We advocate for a national economy based upon innovation and advanced training. In the same way that we were inspired by explorers and scientists when we were young, it is our responsibilty to engage the public and to instill a passion for

\section{KNOWLEDGE}

Following selection (a gratifying day for a fortunate few!), astronauts begin the first phase of our educal vides each new astronaut (no matter what prior professional background) with a common knowledge base and builds the foundation for more advanced raining to follow in the ensuing years. Each new recruit acquires broad background knowledge about the scientific, technical and operational aspects of human spaceflight.

The structure of the basic training program for an astronaut shares a lot in common with a medical school curriculum. Our training resources include manuals, lectures, computer-based instruction and field trips.

The first couple of years of medical schoo were challenging for me due to the massive amount of knowledge that needed to be learned quickly. The same is true in astronautics. Astronaut training is like drinking from a fire hose. The Russian Soyuz and the American Space Shuttle are complex space vehicles. Even more challenging to Understand and operate is the International Space ( mass of 420 tons dimensions that are two times a mass of 20 tons, dim pace equivalent to a four-bedroom house 120 elephone-booth-size racks house spacecreft systems and research experiments. The Station's onboard computers process four million lines of code. A systems approach is used to instruct astronauts about space operations. We consider the composition of our spacecraft to include thermal control, electrical power, life support and many other systems. Just as no system in the human body operates independently, each spacecraft sys-

Physicians and astronauts would be equally handicapped if we could not resort to our unique mnemonics. Acronyms and jargon pepper our conversations with colleagues. I smile to myself whenever I encounter a new space acronym that shares a meaning from my medical past (e.g. ER, D\&C).

SKILLS

Having acquired the fundamentals of spaceflight operations, astronauts next begin a training phase known as Advanced Training. This phase of training is analogous to clinical clerkship and residency since we learn highly specialized paced and fulfilling.

We learn skills that allow us to launch to and return from orbit, to rendezvous and dock with other spacerat, to perform spacewalks (also known as EVAs) and to operate robotic systems such as the Canadarm2. After acquiring proficiency in a skill, we become mentors to the next trainees. 'See one, do one, teach one' is a mantra that also applies to spaceflight.
Practice makes perfect. We train thousands of hours for nominal as well as off-nomina situations. Crew coordination, situational awareness and speed of reaction are critical factors to save our lives, the spacecraft and the mission under contingency situations.

It is during Advanced Training that simulators play a major role. In fact, simulators are the basis for much of our skills acquisition. They are used to prepare astronauts for a variety of flight

For instance, the robotics simulator at the Canadian Space Agency in Montréal uses virtua reality to model Canadarm2, other robotic systems and the Space Station environment. Astronauts velop skils such as Station assembly and the ceapvere

The Neutral Buoyancy Laboratory (NBL) in . basically a huge pool (much larger than an Olympic swimming pool). We exploit water buoyancy to simulate the weightless condition experienced by astronauts during space walks. Small flotation devices or weights are strapped to the space suits

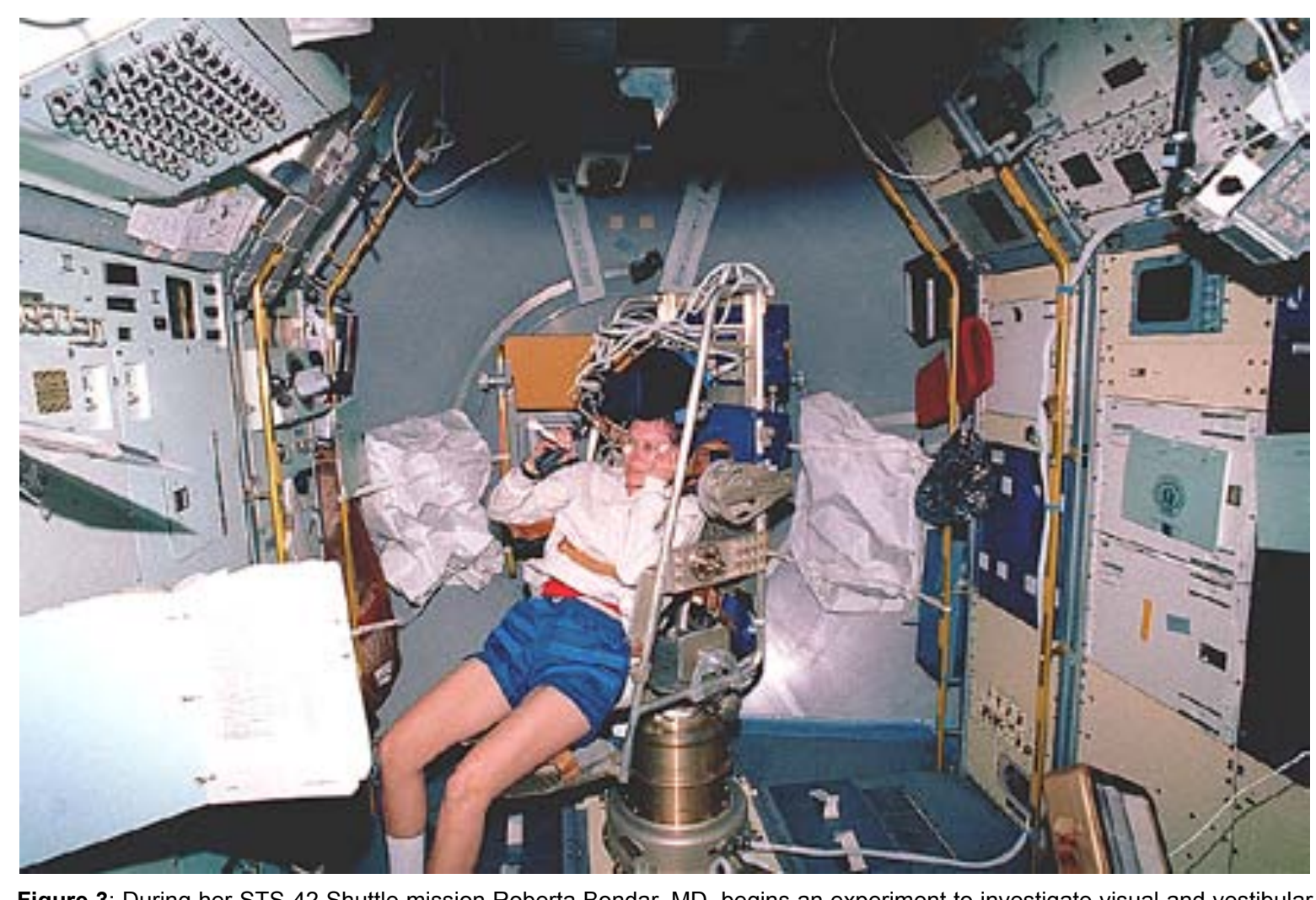

Figure 3: During her STS-42 Shuttle mi 


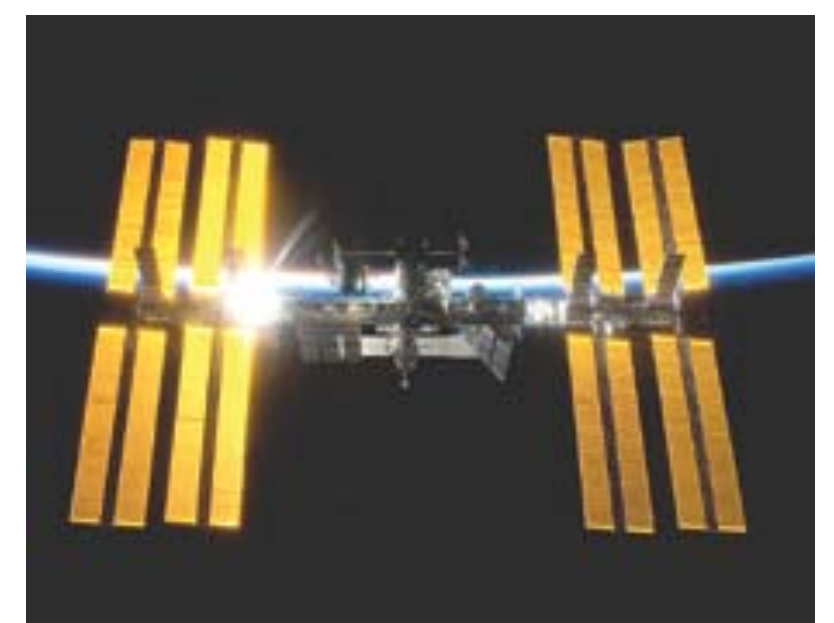
Figure 4: The International Space Station viewed from the
Space Shuttle at orbital sunset.

of astronauts so that we are neutrally buoyant and so that our movements in water are similar to what they would be in space (except for the effect of wamiliarize us with space walk plans and procedures (Figure 5).

The Gagarin Cosmonaut Training Centre near Moscow has an impressive centrifuge with a rotating arm that is 18-meters long (the largest in the world). The distal end of the arm contains a functional mock-up of the Soyuz cockpit. This is where cosmonauts sit. As the arm rotates, the centrifuge creates the g-forces similar to what we experience in our capsule during atmospheric reentry. The level of $g$-force induced by the centrifuge is determined solely by the re-entry profile that
we manually fly from the controls in the cockpit. In we manually fly from the controls in the cockpit. In
other words, we pay for any piloting mistakes we other words, we pay for any piloting mistakes we
may make with a high g-load! Talk about incentive may make with a high g-load!
for the trainee to get it right!

There are many other simulators used for astronaut training and they come in a variety of appearances and functions. No single simulator can each is ablo to simulate one or more features in high fidelity. By integrating our training experiences across all by ine simulars, we are well prepared for what we will encounter on orbit.

Another valuable training resource is NASA's fleet of T-38 high performance jets (Figure 6). These aircraft are not simulators; they provide reallife dynamic training. Jet flight, like spaceflight, involves interaction with complex, data-rich systems in a fast-paced, unforgiving environment. Flight time in high performance jets sharpens decisionmaking skills and crew coordination.

\section{ATTITUDES}

An ISS crew of six possesses all the necessary skills to deal wh any onboard situation. Each crewmember fulfills a certain role and is proficien in several specialized skills (in addition to generic crew skills). For instance, during Expedition 20/21, functioned as a fight engineer, and my specialized responsibilities included medical care for the crew payload science and robotics. While the crew commander had overall responsibility, each crew member played a leadership role for specific aspects of
the expedition.

The harmonious teamwork exhibited by my Shuttle and Station crews is something I fondly reule of duties, each of my crewmates becme in volved in the succesful compltion of others' tasks. We anticipated each other's unspoken needs in the same way that an operating room nurse anticipates the next instrument required by a surgeon. After completing our own tasks, we then looked for opportunities to help our crewmates with theirs. For instance, I would arrive at a worksite aboard the Station and find that someone had already gathered the tools that I would require for my upcoming task. What a team

Everyone helps out with everything. Accordingly, when one crew member successfully completes a complex operation, we all share in the satisfaction. This kind of crew interaction enhances our productivity and makes our activities seem tightly choreographed.

Like all health care practitioners, astronauts are vigilant and precise about everything we do. During spaceflight there is often only one chance to perform a task correctly. The speed of the spacecraft, the constraints of orbital dynamics are

Launch, rendezvous and re-entry are phout the next possible failu ville pre-ocupy our minds while executing engine burns and on-our maneuvers. Monitoring our instruments for system malfunctions is not enough; we also need to anticipate the next failure, its impact and our reaction Most astronauts are not familiar with the clinical precept "Primum Non Nocere" but we do adhere to its intent. When a crewmate begins a critical task, we often admonish her or him with the

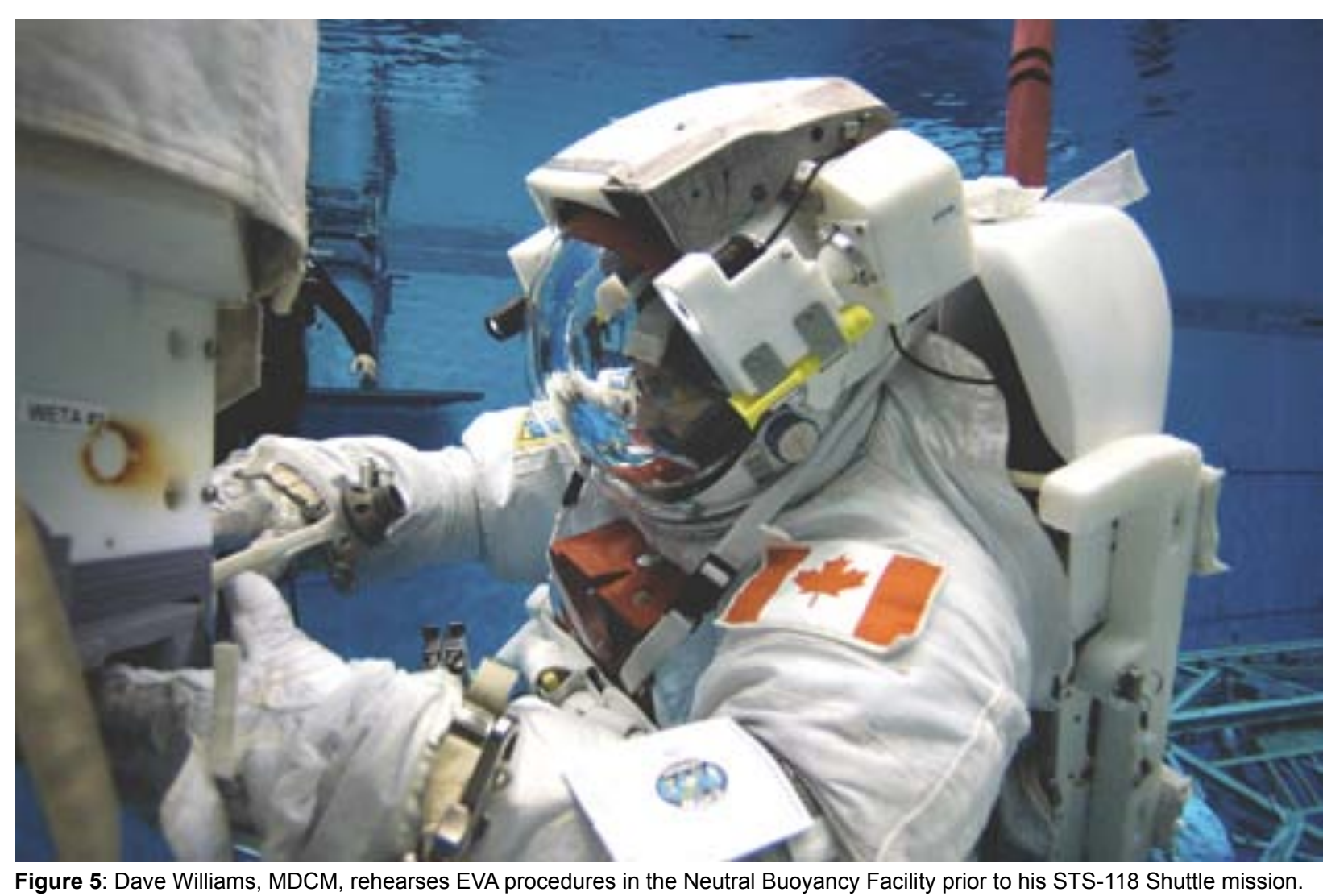

words "don't become famous!" If the name of an astronaut becomes well known, it is often because she or he made a mistake while on-orbit. In the following years, our instructors on the ground will lightheartedy mention the goof-up and our name to forewarn the next classes of potential operational pitfalls. I would be happy to complete my career as an unknown

Doctors do not work in isolation from the rest of the hospital staff. Clerks, orderlies, technicians, social workers, physiotherapists and the omniscient head nurse are key members of the patient care team. Astronauts also do not work in isolation. Thousands of kilometers away at mission control scientists, technians, ers (known collectively as 'flight controllers') continuously monitor our spacecreft's telemetry, video and audio signals. The flight controllers have great insight into the status of our vehicle's systems and payloads, and are ready to spring into action if an in-flight anomaly should to cur.

Anomalies and hardware failures can be expected during the course of every mission. For example, during Expedition 20/21 our oxygen generation and carbon dioxide removal systems failed.
At these times I considered the flight controllers as specialists and regarded my role in space as their eyes, ears, and hands. Oslerian observation and communication skills that I had developed as a physician became useful. Having completed the hisIn examination of the failed system, I then Whe problem. critical together, we successfully repaired these menters members of our team and I have great confidence heir capabilities.

Physicians may be the most senior practithers on be are only vill subsets of large talented teams devoted to success.

A space mission continues even after we return to Earth. Astronauts spend several weeks after landing in medical testing and physical rehabilitation. We don't consider the mission complete until the debriefings are finished.

No mission has ever been flown perfectly. Hardware inevitably breaks down and contingency situations arise. Every astronaut will admit communicated my observations concisely to the ing anter 
in hindsight that she or he could have executed a particular task more effectively. During debriefing sessions (our version of Grand Rounds), we recount our experiences for the benefit of instructors, flight controllers and program managers. This kind of feedback facilitates planning for future missions and enhances the training for the crews who will fly next. We learn and improve from our insights and errors.

\section{CONCLUSION}

Back on Earth, I often reflect on my medical school years and residency. Both were positive experiences. As a student l couldn't foresee the day offer to mat moth medical training would someday offer to me both on and of this planet. to recruit physicians for our unique tining exper-

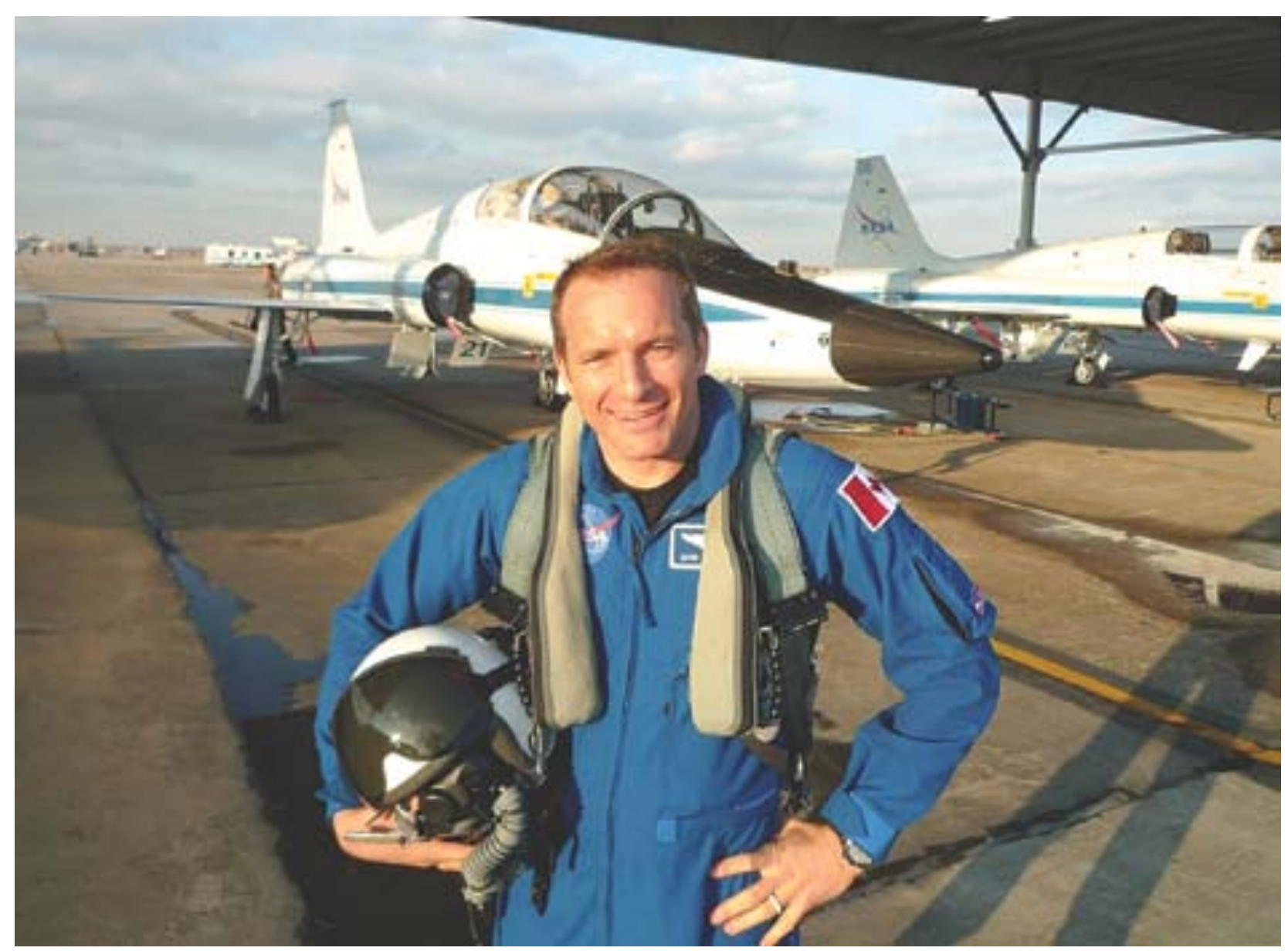

Figure 6: David Saint-Jacques, MD, returns from a training flight in a T-38 jet. These jets develop operator skills and crew coordination Figure 6: David Saint-Jaca
in a dynamic environment.

ences and for the operational capabilities that we hone on hospital wards. Of Canada's ten astronauts, four are physicians. Three of these four phy-

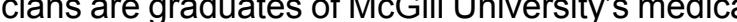
The knot Not a bad batting average! cinician are valuable but not sufficient, of course, to be an astronaut. A burning passion for space exploration is also required. We take our inspiration from John F. Kennedy who, when the United States was initiating its Apollo moon program, declared We choose to go to the moon, not because will seve to mecause it is hard, because that goal whis serve to measure the best of our energies and For some people, the benefits of space exisk. For physician-astronauts, they clearly do.

\section{.}

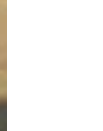
.

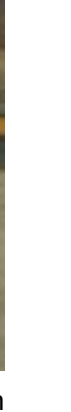

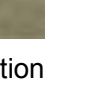

